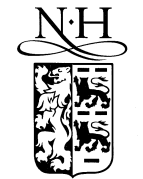

ELSEVIER

\title{
Self modulation phenomena in an external-cavity semiconductor laser
}

\author{
Yih-Jun Wong, Hong-Hsieng Chen, Choong-Wen Lai, Yean-Woei Kiang, \\ C.C. Yang*
}

Department of Electrical Engineering, Graduate Institute of Electro-Optical Engineering, and Graduate Institute of Communication Engineering, National Taiwan University, 1 Roosevelt Road, Sec. 4, Taipei, Taiwan

Received 12 November 1999; received in revised form 7 March 2000; accepted 15 April 2000

\begin{abstract}
We report the observed self-modulation phenomena in an external-cavity semiconductor laser, in which a slant ridge-loading waveguide was formed for the gain medium. A region beside the ridge at a cleaved end of the waveguide served as a saturable absorber for self-modulation. Under different conditions of injection current and cavity alignment, we observed three laser operation regimes, including pure $\mathrm{cw}$ oscillation, constant-amplitude pulse-like signal on the top of a cw component, and amplitude-modulated pulse-like signal coexistent with a $\mathrm{cw}$ component. The amplitude modulation phenomenon is different from the previously reported concurrence of mode-locking and self-pulsation. A model was proposed to explain the observed results. (C) 2000 Published by Elsevier Science B.V. All rights reserved.
\end{abstract}

PACS: 42.55.Px; 42.60.Fc

Keywords: Saturable absorber; Semiconductor lasers

Pulsed signal generation from a laser, particularly from a semiconductor laser, is crucially important for high-speed optical communications. In semiconductor lasers, short pulse generation can be implemented through gain switching, mode-locking, and the combination of the last two methods with the fiber technology [1,2]. Although active mode-locking or hybrid mode-locking leads to more stable pulse trains [3-5], passive mode-locking bears the advantage of

\footnotetext{
* Corresponding author. Tel.: +886-2-2365-7624; fax: +886-22365-2637; e-mail: ccy@cc.ee.ntu.edu.tw
}

simplicity [6,7]. The most commonly used modelocking mechanism for semiconductor laser is absorption saturation. The interplay of absorption saturation in the section of saturable absorber and gain saturation in the section of gain medium can result in the generation of picosecond or shorter pulses. In a semiconductor laser system with a saturable absorber, besides passive mode-locking, self-pulsation was commonly observed [8]. Self-pulsation in semiconductor lasers is similar to passive Q-switching in solid-state lasers. In this mechanism, pulsed laser is generated during absorption saturation of the saturable absorber. Laser is self-switched off when carriers relax and absorption resumes. Because both 
are induced by absorption saturation in the saturable absorber, passive mode-locking and self-pulsation can usually observed simultaneously in a semiconductor laser with a saturable absorber [8,9]. In such a situation, the amplitude of the mode-locked pulse train is modulated, similar to the output of an actively Q-switched, passively mode-locked Nd:YAG laser. Such phenomena were observed in a multiplesection semiconductor laser. They were also observed in a semiconductor laser without designated saturable absorber. In this situation, the regions on the two sides of the ridge-loading waveguides, which receive fewer injection carriers, serve as the saturable absorbers [10].

In this paper, we report our results of self-modulation of an external-cavity semiconductor laser. In this laser system, pulse-like signals are accompanied with $\mathrm{cw}$ components. Carrier concentration evolution in an unintentional saturable absorber leads to the alternating dominance between the pulse-like signal and the $\mathrm{cw}$ component. This phenomenon is different from the aforementioned Q-switched mode-locking case as reported previously. Instead, what we observed represents the self-modulation of the pulse-like signal generation mechanism. It is related to the competition between the pulse-like and cw components. In the discussions below, a model will be proposed to explain the observed phenomena.

Fig. 1 shows the layout of the external-cavity semiconductor laser, in which the semiconductor optical amplifier (SOA) was fabricated with an epitaxial structure of GaAs/AlGaAs multiple quantum wells (three GaAs wells of $6.5 \mathrm{~nm}$ in thickness and two $\mathrm{Al}_{0.25} \mathrm{Ga}_{0.75} \mathrm{As}$ barriers of $20 \mathrm{~nm}$ in thickness)

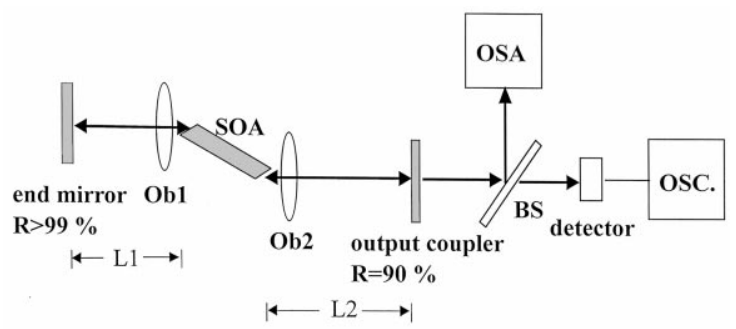

Fig. 1. Layout of the external-cavity semiconductor laser. SOA: semiconductor optical amplifier; Ob1 and Ob2: collimating objectives; OSA: optical spectrum analyzer; BS: beam splitter; OSC.: oscilloscope.

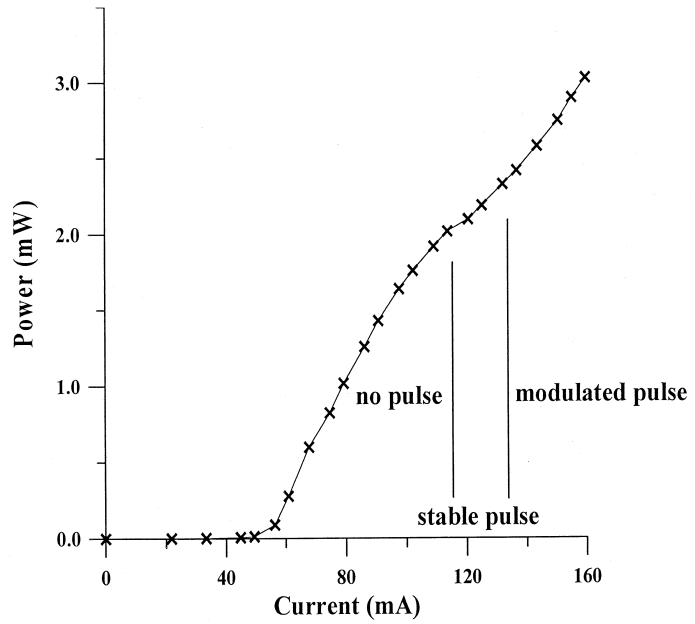

Fig. 2. L-I curve under a set of alignment condition. Three laser operation regimes can be identified.

with the gain peak near $840 \mathrm{~nm}$. The quantum well layers were sandwiched by $0.1 \mu \mathrm{m}$ intrinsic $\mathrm{Al}_{0.25} \mathrm{Ga}_{0.75}$ As separate confinement layer on each side. The $\mathrm{p} / \mathrm{n}$-type $\mathrm{Al}_{0.4} \mathrm{Ga}_{0.6}$ As claddings were 1.6 $\mu \mathrm{m}$ in thickness. On the top, a p-type GaAs layer of $0.1 \mu \mathrm{m}$ was grown for protection. A slant ridge-loading waveguide of $4 \mu \mathrm{m}$ in width was formed with an angle of 12 degrees between the waveguide axis and the normal of the cleaved facet. This angle was designed to reduce the end-facet reflectivity down below $10^{-6}$ so that multiple-reflection would not occur in the external-cavity laser. The ridge-loading waveguide was formed by etching a depth of about 1 $\mu \mathrm{m}$. The lengths of the external cavities on the two sides could be arbitrarily chosen.

A set of L-I data is shown in Fig. 2, from which the threshold current at $55 \mathrm{~mA}$ can be seen. With the injection current between 55 and $115 \mathrm{~mA}$, pure $\mathrm{cw}$ laser output was observed. After the kink around 115 $\mathrm{mA}$, pulse-like trains were observed. At a fixed injection current between 115 and $135 \mathrm{~mA}$, the pulse-like train has a constant amplitude, accompanied with a constant $\mathrm{cw}$ component. However, beyond $135 \mathrm{~mA}$ self-modulation of the pulse-like component could be observed. Note that the transitions between the three regimes were not abrupt. The aforementioned current values for dividing the three regimes are the estimated values. Fig. 3 shows a pulse-like train of constant pulse and cw components 


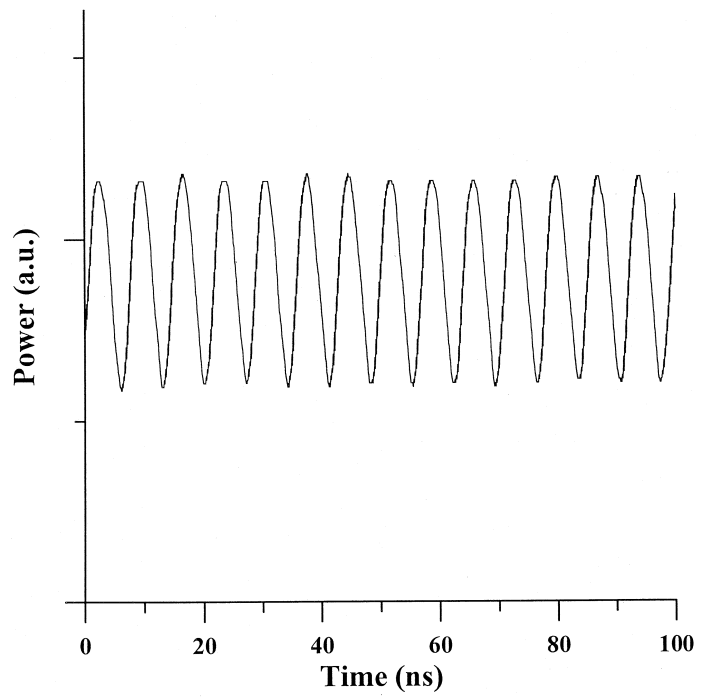

Fig. 3. A steady pulse-like train in the situation of constant-amplitude pulse-like and cw components coexistent.

when the injection current is $125 \mathrm{~mA}$. The pulse-like repetition rate at $150 \mathrm{MHz}$ is the same as the free spectral range of the resonance cavity, whose length is $100 \mathrm{~cm}$ in this case. Hence, the pulse-like signal generation can be attributed to mode-locking or mode-beating. Unfortunately, no autocorrelation trace was obtained for confirming mode-locking. The nearly sinusoidal pattern (actually not sinusoidal) can be attributed to the slow responses of the used photodetector (200 ps response time) and oscilloscope $(500 \mathrm{MHz})$. Fig. 4 shows a result of amplitude modulation of the pulse-like component when the injection current is increased to $153 \mathrm{~mA}$. Here, one can see that the pulse-like component is modulated with a frequency of about $20 \mathrm{MHz}$. This modulation is different from those previously reported [11]. Previously, amplitude modulations with lower frequencies of pulses were reported. However, the result shown in Fig. 4 describes the periodical dominance of the pulse-like and cw components.

In Fig. 5, three curves are plotted for the output spectra recorded when the injection current was 111 (pure cw output), 125 (constant-amplitude pulse-like and $\mathrm{cw}$ components coexistent), and $153 \mathrm{~mA}$ (modulated pulse-like and $\mathrm{cw}$ components coexistent), respectively. One can first notice the well-known trend of red-shift with increasing injection current.
Also, the spectrum is broadened when the pulse-like component appears. The secondary humps here may contribute to the $\mathrm{cw}$ components. Note that the primary humps have a width of about $3 \mathrm{~nm}$. Since the cavity length of 1-m in order of magnitude corresponds to a modal spacing of a few tenths of pm, more than 10000 modes exist in the laser output.

The observed phenomena can be explained with the model shown in Fig. 6, in which the possible light beam end-fire coupling scenarios into the slant ridge-loading waveguide are depicted. It is noted that with our $40 \times$ objectives for focusing the feedback light beam, the focal spot size cannot be smaller than $20 \mu \mathrm{m}$. Therefore, the $4 \mu \mathrm{m}$ wide guiding region of the waveguide cannot accept all the incoming power. The shaded ellipse in the figure represents the focused light spot, which distributes part of power outside the ridge-loading region. This region, denoted by SA, can serve as a saturable absorber for this part of power before it eventually (at least partially) couples into the waveguide for gain. In other words, this laser system consists of two simultaneous oscillating components. The part of power directly coupled into the ridge-loading region (denoted by A) oscillates to produce the $\mathrm{cw}$ component. On the other hand, the oscillation of the part coupled into the SA region is responsible for the pulse-like

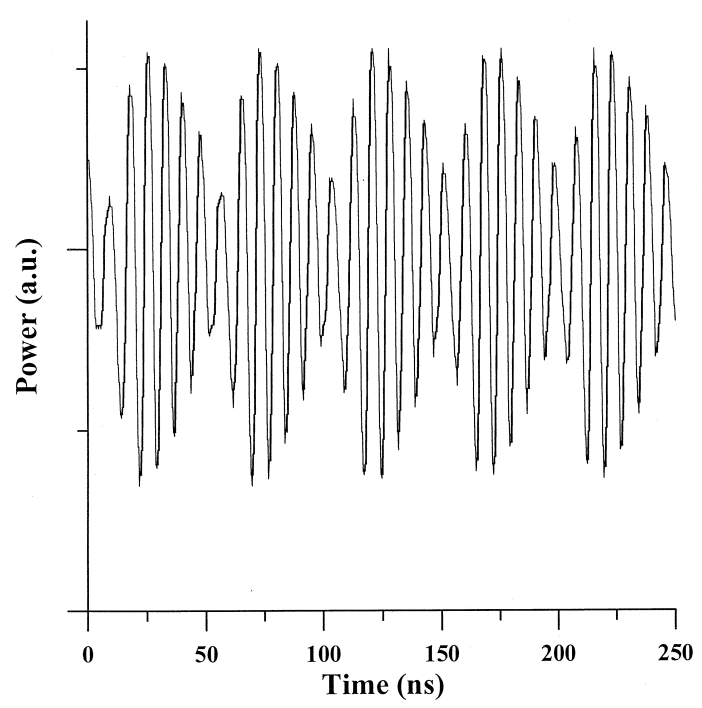

Fig. 4. A pulse-like train in the situation of modulated pulse-like and $\mathrm{cw}$ components coexistent. 


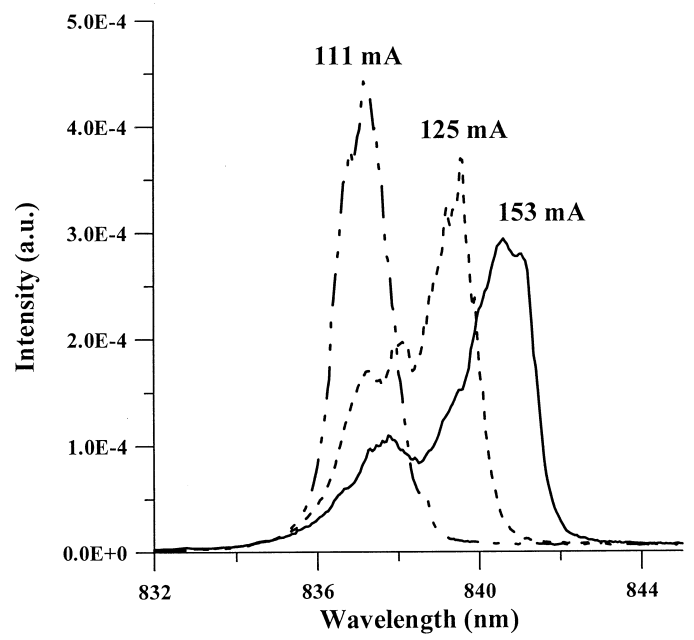

Fig. 5. Spectra of three laser outputs corresponding to the three laser operation regimes.

component. These two components may compete for dominating the laser oscillation. In the injection current range between 115 and $135 \mathrm{~mA}$, a balance is reached. However, as the current increases, more carrier supply leads to the possibility of complete absorption saturation in the SA region. When absorption in the SA region is deeply saturated, the pulselike signal generation mechanism is suppressed, leading to a stronger $\mathrm{cw}$ component. After carriers are relaxed, this mechanism resumes its operation. Such a periodical process results in the modulated pulselike signal as shown in Fig. 4. A close look at the modulation in Fig. 4 reveals the relatively faster recovery of the pulse-like signal generation mechanism, compared with its decay. This indicates that carrier relaxation in the SA region may be faster than band filling. Since the modulation frequency is at the order of few tens $\mathrm{MHz}$, carrier dynamics involved in the aforementioned process may be related to carrier diffusion besides carrier recombination. However, this claim requires further confirmation.

From the discussions above, one can see that the laser operation is determined by the location and size of the feedback light beam. Meanwhile, they are controlled by the orientations of the end mirror and output coupler and the positions of the two collimating objectives. The variation of alignment conditions results in various modulation results. In one of the tests, we fixed the light beam location in the direc- tion of material growth and moved the beam laterally. Under the condition of a fixed injection current, we observed the consecutive results of no laser, pure $\mathrm{cw}$ laser, constant-amplitude pulse-like and cw components coexistent, modulated pulse-like and $\mathrm{cW}$ components coexistent, and no laser again as the feedback light beam moved from the bottom to top in Fig. 6. Such variations of laser output assure the validity of the proposed model. We also varied the ratio of the lengths of the two external cavities (fixed total cavity length) and found no significant changes of the results in pulse-like repetition rate and modulation frequency. This excludes the possible mechanism of pulse colliding in our laser system. Recently, stable mode-locked pulses from a ring-configuration external-cavity semiconductor laser with a slant ridge waveguide were observed [12]. The mode-locking mechanism was proposed to be self-bending at the edge of the slant waveguide. However, our results cannot be attributed to self-bending since no pulselike results were observed when we replaced ridgeloading waveguides with ridge waveguides (etching depth $1.8 \mu \mathrm{m}$ ). In other words, when we etched away the active layer in the regions outside the waveguide (to form a ridge waveguide), only $\mathrm{cw}$ laser could be implemented. In this situation, pulselike signals and their self-modulation could not be observed.

In summary, we have observed self-modulation of pulse-like signal generation mechanism in an external-cavity semiconductor laser. The regions outside the ridge at the cleaved ends of the slant ridge-loading waveguide served as saturable absorbers for pulse-like signal generation and self modulation.

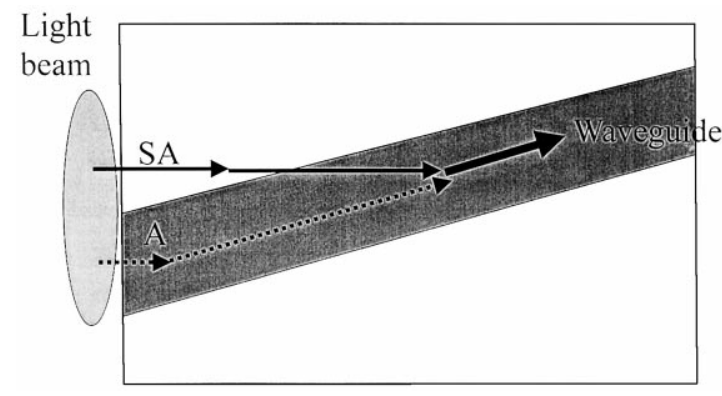

Fig. 6. Model used to explain the self-modulation of the pulse-like signal generation mechanism. SA: saturable absorber region; A: active region with gain. 
With different laser system conditions, including injection current and cavity alignment, three laser operation regimes could be realized. They included pure $\mathrm{cw}$ oscillation, constant-amplitude pulse-like and cw components coexistent, and modulated pulse-like and $\mathrm{cw}$ components coexistent. A model based on the variation of feedback beam coupling situation was proposed to explain well the observed phenomena. Although we could not assure whether modelocking was achieved so far, we did observe the novel phenomena of self-modulation of pulse-like signal generation mechanism. Further investigations include the improvement of the laser system and the measurement arrangement for assuring mode-locking. A better control of the light beam coupling into the waveguide for suppressing the $\mathrm{cw}$ component is also interesting.

\section{Acknowledgements}

The support of this research from The National Science Council, Republic of China, under the grants NSC 2215-E-002-012, NSC 88-2112-M-002-004, and NSC 88-2215-E-002-014 are acknowledged.

\section{References}

[1] S. Li, K.T. Chan, Y. Liu, L. Zhang, I. Bennion, Multiwavelength picosecond pulses generated from a self-seeded Fabry-Perot laser diode with a fiber external cavity using fiber Bragg gratings, IEEE Photon. Technol. Lett. 10 (1998) 1712-1714.

[2] K. Chan, C. Shu, Electrically wavelength-tunable picosecond pulses generated from a self-seeded laser diode using a compensated dispersion-tuning approach, IEEE Photon. Technol. Lett. 11 (1999) 1093-1095.

[3] J.E. Bowers, P.A. Morton, A. Mar, S.W. Corzine, Actively mode-locked semiconductor lasers, IEEE J. Quantum Electron. 25 (1989) 1426-1439.

[4] M. Shell, A.G. Weber, E. Scholl, D. Bimberg, Fundamental limits of sub-ps pulse generation by active mode-locking of semiconductor lasers: the spectral gain width and the facet reflectivities, IEEE J. Quantum Electron. 27 (1991) 16611667.

[5] S. Arahira, Y. Ogawa, Passive and hybrid mode-locking in a multi-electrode DBR laser with two gain sections, Electron. Lett. 31 (1995) 808-809.

[6] Y.K. Chen, M.C. Wu, Monolithic colliding-pulse modelocked quantum-well lasers, IEEE J. Quantum Electron. 28 (1992) 2176-2185.

[7] J.P. Hohimer, G.A. Vawter, Passive mode-locking of monolithic semiconductor ring lasers at $86 \mathrm{GHz}$, Appl. Phys. Lett. 63 (1993) 1598-1603.

[8] S. Sanders, L. Eng, A. Yariv, Passive mode-locking of monolithic InGaAs / AlGaAs double quantum well lasers at $42 \mathrm{GHz}$ repetition rate, Electron. Lett. 25 (1989) 1087-1089.

[9] Vasil'ev, Ultrafast Diode Lasers, Artech House, 1995.

[10] M. Yamada, A theoretical analysis of self-sustained pulsation phenomena in narrow-stripe semiconductor lasers, IEEE J. Quantum Electron. 29 (1993) 1330-1336.

[11] W. Yang, A. Gopinath, Study of passive mode locking of semiconductor lasers using time-domain modeling, Appl. Phys. Lett. 63 (1993) 2717-2719.

[12] P. Langlois, M. Piche, Self-mode-locked Semiconductor Laser in a Ring Cavity, Paper CPD1.3, The 1998 European Conference on Lasers and Electro-Optics, October, Glasgow, UK, 1998. 